\title{
Editorial
}

\section{Antimicrobial Peptides as Mediators of Innate Immunity}

In this special issue of Current Pharmaceutical Design, entitled: “Antimicrobial peptides as mediators of innate immunity”, the major features of Antimicrobial Peptides (AMPs) are outlined. In particular, their classification, cellular sources, and biological properties in health and disease are described. Finally, clinical applications of AMPs, as novel therapeutics in the course of infections caused by antibioticresistant bacteria, fungi, protozoa and viruses as well as in cancer, are discussed.

Magrone et al. [1] at first review the phylogenic sources of AMPs as well as their anti-inflammatory and immunomodulating activities. Moreover, lantibiotics, as new potential anti-infective drugs, are described. Authors also point out the ability of dietary bioactive principles and food supplements to induce AMP production.

Pizzo et al. [2] place emphasis on the exploitation of structure, function and mechanisms of action exerted by AMPs in drug targeting. In this review, cryptic AMPs are introduced as proteins hidden in their primary structure and, then, released by the host and/or bacterium proteases. Their discovery has enlarged the spectrum of known AMPs, also allowing investigation of new functions and applications.

Drago-Serrano et al. [3] describe the role of Lactoferrin (LF) and lactoferricins (Lfcins) in the control of pathogens also in virtue of their immune regulatory properties. LF and Lfcins are molecules of pharmacological interest and native LF and its N-terminus peptides seem to have potential applications as adjunctive anti-infectious agents. Finally, these compounds have the property to retard tumor growth and attenuate endotoxin shock.

Chieosilapatham et al. [4] review the role played by human cathelicidin LL-37 in the modulation of innate immune response. Particularly, LL-37 exerts pro- and anti-inflammatory activities, also inducing maturation, proliferation and regulation of apoptosis. Finally, according to its anatomical distribution, LL-37 seems to play different roles in the regulation of innate immunity.

Kiatsurayanon et al. [5] illustrate the role of human beta-defensins (hBDs) in the maintenance of skin barriers. They exert a broad spectrum of microbicidal activities also displaying several immunoregulatory activities in the course of skin infections and inflammation. A better knowledge of hBD physiology may contribute to develop new therapeutic remedies in the course of skin barrier impairment, such as atopic dermatitis and psoriasis.

Skovbakke et al. [6] outline the property of Formyl Peptide Receptors (FPRs) in mediating the immunomodulatory activities of AMPs and peptidomimetics. In this framework, for their function neutrophils express FPRs which behave as targets of AMPs. On these bases, peptidomimetics represent new molecules able to modulate neutrophil activities.

Adolph et al. [7] describe the function of intestinal Paneth cells as producers of AMPs finalized to the homeostatic control of microbiota. Emphasis is placed on the alteration of Paneth cell function in inflammatory disease and, especially, in the course of ulcerative colitis and Crohn's disease.

Khurshid et al. [8] point out the unique structure of human defensins based on amino acid sequences bearing disulphide bridges which allow their synthesis or natural production with the help of bacteria. In particular, in this review, the role exerted by oral defensins in health and disease is stressed out.

Rivas-Santiago and Torres-Juarez [9] highlight the ability of AMPs to kill mycobacteria both in vitro and in vivo. Then, AMPs can be used as an adjunctive therapy in the course of human tuberculosis. However, to avoid collateral effects depending on the multiple activities exerted by AMPs, Authors suggest that certain features, such as comorbidity, family history and risk factors in patients with tuberculosis should be taken into consideration prior to AMP therapy start.

Magrone et al. [10] emphasize the concept that AMPs in comparison to antibiotics possess a larger spectrum of antimicrobial activities without inducing bacterial resistance. Therefore, their use in different clinical settings, even including septic shock is discussed. Finally, a series of novel compounds derived from AMPs for their potential capacity to reinforce the immune response are illustrated. 


\section{REFERENCES}

[1] Magrone T, Russo MA, Jirillo E. Antimicrobial peptides: Phylogenic sources and biological activities. First of Two Parts. Curr Pharm Des 2018; 24(10): 1043-53.

[2] Pizzo E, Cafaro V, Di Donato A, Notomista E. Cryptic Antimicrobial Peptides: Identification methods and current knowledge of their immunomodulatory properties. Curr Pharm Des 2018; 24(10): 1054-66.

[3] Drago-Serrano ME, Campos-Rodríguez R, Carrero JC, de la Garza M. Lactoferrin and peptide-derivatives: Antimicrobial agents with potential use in nonspecific immunity modulation. Curr Pharm Des 2018; 24(10): 1067-78.

[4] Chieosilapatham P, Ikeda S, Ogawa H, Niyonsaba F. Tissue-specific regulation of innate immune responses by human cathelicidin LL-37. Curr Pharm Des 2018; 24(10): 1079-91.

[5] Kiatsurayanon C, Ogawa H, Niyonsaba F. The role of host defense peptide human $\beta$-defensins in the maintenance of skin barriers. Curr Pharm Des 2018; 24(10): 1092-9.

[6] Skovbakke SL, Holdfeldt A, Forsman H, Bylund J, Franzyk H. The role of formyl peptide receptors for immunomodulatory activities of antimicrobial peptides and peptidomimetics. Curr Pharm Des 2018; 24(10): 1100-20.

[7] Adolph TE, Mayr L, Grabherr F, Tilg H. Paneth cells and their antimicrobials in intestinal immunity. Curr Pharm Des 2018; 24(10): 1121-9.

[8] Khurshid Z, Zafar MS, Naseem M, Khan RS, Najeeb S. Human oral defensins antimicrobial peptides: A future promising antimicrobial drug. Curr Pharm Des 2018; 24(10): 1130-7.

[9] Rivas-Santiago B, Torres-Juarez F. Antimicrobial peptides for the treatment of pulmonary tuberculosis, allies or foes? Curr Pharm Des 2018; 24(10): $1138-47$.

[10] Magrone T, Russo MA, Jirillo E. Antimicrobial peptides in human disease: Therapeutic approaches. Second of Two Parts. Curr Pharm Des 2018; 24(10): 1148-56.

Jirillo Emilio and Thea Magrone

Department of Basic Medical Sciences, Neuroscience and Sensory Organs, University of Bari, School of Medicine, 